\title{
El Gamal Cryptosystem on a Montgomery Curves Over Non Local Ring
}

\author{
${ }^{1}$ MOHA BEN TALEB ELHAMAM, ${ }^{1}$ ABDELALI GRINI, ${ }^{2}$ ABDELHAKIM CHILLALI, \\ ${ }^{1}$ LHOUSSAIN EL FADIL \\ Department of Mathematics \\ ${ }^{1}$ Sidi Mohamed Ben Abdellah University, Faculty of Science Dhar El Mahraz \\ ${ }^{2}$ Sidi Mohamed Ben Abdellah University, FP, LSI, Taza \\ Atlas, Fez, postcode 1796, Fez, Morocco \\ MOROCCO
}

Abstract: Let $\mathbb{F}_{q}$ be the finite field of $q$ elements, where $q$ is a prime power. In this paper, we study the Montgomery curves over the ring $\frac{\mathbb{F}_{q}[X]}{\left\langle X^{2}-X\right\rangle}$, denoted by $M_{A, B}\left(\frac{\mathbb{F}_{q}[X]}{\left\langle X^{2}-X\right\rangle}\right) ;(A, B) \in\left(\frac{\mathbb{F}_{q}[X]}{\left\langle X^{2}-X\right\rangle}\right)^{2}$.

Using the Montgomery equation, we define the Montgomery curves $M_{A, B}\left(\frac{\mathbb{F}_{q}[X]}{\left\langle X^{2}-X\right\rangle}\right)$ and we give a bijection between this curve and product of two Montgomery curves defined on $\mathbb{F}_{q}$. Furthermore, we study the addition law of Montgomery curves over the ring $\frac{\mathbb{F}_{q}[X]}{\left\langle X^{2}-X\right\rangle}$. We close this paper by introducing a public key cryptosystem which is a variant of the ElGamal cryptosystem on a Montgomery curves over the same ring.

Key-Words: Montgomery curves, Finite ring, Cryptography, ElGamal.

Received: May 10, 2021. Revised: January 13, 2022. Accepted: February 8, 2022. Published: March 2, 2022.

\section{Introduction}

Let $\mathbb{F}_{q}$ be the finite field of order $q=p^{n}$ where $n$ is a positive integer and $p$ is a prime number. The ring $\frac{\mathbb{F}_{q}[X]}{\left\langle X^{2}-X\right\rangle}$ can be identified to the finite ring $\mathbb{F}_{q}[e], e^{2}=e$. The objective of this article is the search for new groups of points of a Montgomery curve on a finite ring, for use in cryptography. In [10, Montgomery introduced a new elliptic curve model what became known as Montgomery curves and the Montgomery scale as way to speed up Lenstra's elliptic-curve factorization method [8]. Boulbot et al. study the arithmetic of the ring $\mathbb{F}_{q}[e]$, in particular they show that this ring is not a local 2. In section 3 , we define the Montgomery curves $M_{A, B}\left(\mathbb{F}_{q}[e]\right)$ over this ring, we study Montgomery equation which allows us to define two Montgomery curves: $M_{\pi_{0}(A), \pi_{0}(B)}\left(\mathbb{F}_{q}\right)$ and $M_{\pi_{1}(A), \pi_{1}(B)}\left(\mathbb{F}_{q}\right)$ defined over the finite field $\mathbb{F}_{q}$. In the next of this section, we classify the elements of $M_{A, B}\left(\mathbb{F}_{q}[e]\right)$ and we give a bijection between the two sets: $M_{A, B}\left(\mathbb{F}_{q}[e]\right)$ and $M_{\pi_{0}(A), \pi_{0}(B)}\left(\mathbb{F}_{q}\right) \times$ $M_{\pi_{1}(A), \pi_{1}(B)}\left(\mathbb{F}_{q}\right)$, where $\pi_{0}$ and $\pi_{1}$ are two surjective morphisms of rings defined by:

$$
\begin{aligned}
\pi_{0}: \mathbb{F}_{q}[e] & \rightarrow \mathbb{F}_{q} \\
x_{0}+x_{1} e & \mapsto x_{0}
\end{aligned}
$$

and

$$
\begin{aligned}
\pi_{1}: \mathbb{F}_{q}[e] & \rightarrow \quad \mathbb{F}_{q} \\
x_{0}+x_{1} e & \mapsto x_{0}+x_{1} .
\end{aligned}
$$

We study the addition law of Montgomery curves over the ring $\mathbb{F}_{q}[e]$. We finish this paper by introducing a new public key cryptosystem which is a variant of the ElGamal cryptosystem 3 on a Montgomery curves over the ring $\mathbb{F}_{q}[e]$. For more works in this direction we refer the reader to [1].

\section{The ring $\mathbb{F}_{q}[e], e^{2}=e$}

An element in $\mathbb{F}_{q}[e]$ is represented by $x_{0}+x_{1} e$ where $\left(x_{0}, x_{1}\right) \in \mathbb{F}_{q}$.

The arithmetic operations in $\mathbb{F}_{q}[e]$ can be decomposed into operations in $\mathbb{F}_{q}$ and they are computed as follows:

$$
\begin{gathered}
X+Y=\left(x_{0}+y_{0}\right)+\left(x_{1}+y_{1}\right) e \\
X . Y=\left(x_{0} y_{0}\right)+\left(x_{0} y_{1}+x_{1} y_{0}+x_{1} y_{1}\right) e,
\end{gathered}
$$

where $X=x_{0}+x_{1} e$ and $Y=y_{0}+y_{1} e$. Let us recall the following results [1, 2]:

- $\left(\mathbb{F}_{q}[e],+,.\right)$ is a finite unitary commutative ring.

- $\mathbb{F}_{q}[e]$ is an $\mathbb{F}_{q}$-vector space of dimension 2 with $\mathbb{F}_{q}$-basis $\{1, e\}$.

- $X . Y=\left(x_{0} y_{0}\right)+\left(\left(x_{0}+x_{1}\right)\left(y_{0}+y_{1}\right)-x_{0} y_{0}\right) e$.

- $X^{2}=x_{0}^{2}+\left(\left(x_{0}+x_{1}\right)^{2}-x_{0}^{2}\right) e$.

- $X^{3}=x_{0}^{3}+\left(\left(x_{0}+x_{1}\right)^{3}-x_{0}^{3}\right) e$. 
- Let $X=x_{0}+x_{1} e \in \mathbb{F}_{q}[e]$, then $X \in\left(\mathbb{F}_{q}[e]\right)^{\times}$(the multiplicative group of $\left.\mathbb{F}_{q}[e]\right)$ if and only if $x_{0} \neq 0$ and $x_{0}+x_{1} \neq 0$. The inverse is given by:

$$
X^{-1}=x_{0}^{-1}+\left(\left(x_{0}+x_{1}\right)^{-1}-x_{0}^{-1}\right) e .
$$

- Let $X \in \mathbb{F}_{q}[e]$, then $X$ is not invertible if and only if $X=x e$ or $X=x-x e$, such that $x \in \mathbb{F}_{q}$.

- $\mathbb{F}_{q}[e]$ is a non local ring.

- $\pi_{0}$ and $\pi_{1}$ are two surjective morphisms of rings.

\section{Montgomery curves over the ring $\mathbb{F}_{q}[e], e^{2}=e$}

In this section, the elements $X, Y, Z, A$ and $B$ are in the ring $\mathbb{F}_{q}[e]$ such that $X=x_{0}+x_{1} e, Y=y_{0}+y_{1} e$, $Z=z_{0}+z_{1} e, A=A_{0}+A_{1} e$ and $B=B_{0}+B_{1} e$ where $x_{0}, x_{1}, y_{0}, y_{1}, z_{0}, z_{1}, A_{0}, A_{1}, B_{0}$ and $B_{1}$ are in $\mathbb{F}_{q}$. We define a Montgomery curve over the ring $\mathbb{F}_{q}[e]$, as a curve in the projective space $P^{2}\left(\mathbb{F}_{q}[e]\right)$, which is given by the equation:

$$
B Y^{2} Z=X^{3}+A X^{2} Z+X Z^{2},
$$

where $A$ and $B$ are parameters satisfying the condition that $\Delta=B\left(A^{2}-4\right)$ is invertible in $\mathbb{F}_{q}[e]$.

We denote this curves by: $M_{A, B}\left(\mathbb{F}_{q}[e]\right)$, and we write:

$$
\begin{gathered}
M_{A, B}\left(\mathbb{F}_{q}[e]\right)=\left\{[X: Y: Z] \in P^{2}\left(\mathbb{F}_{q}\right) \mid B Y^{2} Z=X^{3}+\right. \\
\left.A X^{2} Z+X Z^{2}\right\},
\end{gathered}
$$

there is a unique point $O=[0: 1: 0]$ at infnity in $M_{A, B}$ : it is the only point on $M_{A, B}$ where $Z=0$.

Proposition 1. Let $\Delta_{0}=B_{0}\left(A_{0}^{2}-4\right)$ and $\Delta_{1}=\left(B_{0}+\right.$ $\left.B_{1}\right)\left(\left(A_{0}+A_{1}\right)^{2}-4\right)$. Then,

$$
\Delta=\Delta_{0}+\left(\Delta_{1}-\Delta_{0}\right) e .
$$

Proof. We have:

$$
\begin{aligned}
\Delta & =B\left(A^{2}-4\right) \\
& =\left(B_{0}+B_{1} e\right)\left[\left(A_{0}+A_{1} e\right)^{2}-4\right] \\
& =\Delta_{0}+\left(\Delta_{1}-\Delta_{0}\right) e .
\end{aligned}
$$

Corollary 1. $\Delta$ is invertible in $\mathbb{F}_{q}[e]$ if and only if $\Delta_{0} \neq 0$ and $\Delta_{1} \neq 0$.
Using Corollary 1 if $\Delta$ is invertible in $\mathbb{F}_{q}[e]$, then $M_{\pi_{0}(A), \pi_{0}(B)}\left(\mathbb{F}_{q}\right)$ and $M_{\pi_{1}(A), \pi_{1}(B)}\left(\mathbb{F}_{q}\right)$ are two projective Montgomery curves over the finite field $\mathbb{F}_{q}$, and we notice:

$$
\begin{aligned}
M_{\pi_{0}(A), \pi_{0}(B)}\left(\mathbb{F}_{q}\right)= & \left\{[x: y: z] \in P^{2}\left(\mathbb{F}_{q}\right) \mid B_{0} y^{2} z=x^{3}\right. \\
+ & \left.A_{0} x^{2} z+x z^{2}\right\} \\
M_{\pi_{1}(A), \pi_{1}(B)}\left(\mathbb{F}_{q}\right)= & \left\{[x: y: z] \in P^{2}\left(\mathbb{F}_{q}\right) \mid\left(B_{0}+B_{1}\right)\right. \\
& \left.y^{2} z=x^{3}+\left(A_{0}+A_{1}\right) x^{2} z+x z^{2}\right\}
\end{aligned}
$$

In 2] Boulbot et al. have showed the following proposition:

Proposition 2. Let $X, Y$ and $Z$ in $\mathbb{F}_{q}[e]$, then $[X: Y$ : $Z] \in P^{2}\left(\mathbb{F}_{q}[e]\right)$ if and only if $\left[\pi_{i}(X): \pi_{i}(Y): \pi_{i}(Z)\right] \in$ $P^{2}\left(\mathbb{F}_{q}\right)$, where $i \in\{0,1\}$.

Theorem 2. Let $X, Y$ and $Z$ be in $\mathbb{F}_{q}[e]$, then $[X: Y: Z] \in M_{A, B}\left(\mathbb{F}_{q}[e]\right)$ if and only if $\left[\pi_{i}(X): \pi_{i}(Y):\right.$ $\left.\pi_{i}(Z)\right] \in M_{\pi_{i}(A), \pi_{i}(B)}\left(\mathbb{F}_{q}\right)$, for $i \in\{0,1\}$.

Proof. We have:

$$
\begin{aligned}
B Y^{2} Z= & \left(B_{0}+B_{1} e\right)\left(y_{0}+y_{1} e\right)^{2}\left(z_{0}+z_{1} e\right) \\
= & B_{0} y_{0}^{2} z_{0}+\left[\left(B_{0}+B_{1}\right)\left(y_{0}+y_{1}\right)^{2}\left(z_{0}+z_{1}\right)-B_{0}\right. \\
& \left.y_{0}^{2} z_{0}\right] e \\
X^{3}= & \left(x_{0}+x_{1} e\right)^{3} \\
= & x_{0}^{3}+\left[\left(x_{0}+x_{1}\right)^{3}-x_{0}^{3}\right] e \\
A X^{2} Z= & \left(A_{0}+A_{1} e\right)\left(x_{0}+x_{1} e\right)^{2}\left(z_{0}+z_{1} e\right) \\
= & A_{0} x_{0}^{2} z_{0}+\left[\left(A_{0}+B_{1}\right)\left(x_{0}+x_{1}\right)^{2}\left(z_{0}+z_{1}\right)-A_{0}\right. \\
& \left.x_{0}^{2} z_{0}\right] e \\
X Z^{2}= & \left(x_{0}+x_{1} e\right)\left(z_{0}+z_{1} e\right)^{2} \\
= & x_{0} z_{0}^{2}+\left[\left(x_{0}+x_{1}\right)\left(z_{0}+z_{1}\right)^{2}-x_{0} z_{0}^{2}\right] e .
\end{aligned}
$$

As $\{1, e\}$ is an $\mathbb{F}_{q}$-basis of the vector space $\mathbb{F}_{q}[e]$,

then: $B Y^{2} Z=X^{3}+A X^{2} Z+X Z^{2}$ if and only if

$$
\left\{\begin{array}{l}
B_{0} y_{0}^{2} z_{0}=x_{0}^{3}+A_{0} x_{0}^{2} z_{0}+x_{0} z_{0}^{2} \\
\text { and } \\
\left(B_{0}+B_{0}\right)\left(y_{0}+y_{1}\right)^{2}\left(z_{0}+z_{1}\right)=\left(x_{0}+x_{1}\right)^{3}+\left(A_{0}+\right. \\
\left.A_{1}\right)\left(x_{0}+x_{1}\right)^{2}\left(z_{0}+z_{1}\right)+\left(x_{0}+x_{1}\right)\left(z_{0}+z_{1}\right)^{2},
\end{array}\right.
$$

so the point $[X: Y: Z]$ is a solution of the Montgomery equation in $M_{A, B}\left(\mathbb{F}_{q}[e]\right)$ if and only if $\left[\pi_{i}(X): \pi_{i}(Y): \pi_{i}(Z)\right]$ is a solution of the same equation in $M_{\pi_{i}(A), \pi_{i}(B)}\left(\mathbb{F}_{q}\right)$ where $i \in\{0,1\}$.

From the Corollary 1 and Proposition 2 we deduce the result.

Corollary 3 . The mappings $\tilde{\pi_{0}}$ and $\tilde{\pi_{1}}$ are well defined, where $\tilde{\pi}_{i}$ for $i \in\{0,1\}$ is given by:

$$
\begin{aligned}
& \tilde{\pi}_{i}: M_{A, B}\left(\mathbb{F}_{q}[e]\right) \rightarrow M_{\pi_{i}(A), \pi_{i}(B)}\left(\mathbb{F}_{q}\right) \\
& {[X: Y: Z] \mapsto\left[\pi_{i}(X): \pi_{i}(Y): \pi_{i}(Z)\right]}
\end{aligned}
$$


Proof. From the previous theorem, we have $\left[\pi_{i}(X): \pi_{i}(Y): \pi_{i}(Z)\right] \in M_{\pi_{i}(A), \pi_{i}(B)}\left(\mathbb{F}_{q}\right)$

If $\left[X_{1}: Y_{1}: Z_{1}\right]=\left[X_{2}: Y_{2}: Z_{2}\right]$, then there exist $\gamma \in$ $\left(\mathbb{F}_{q}\right)^{\times}$such that: $X_{2}=\gamma X_{1}, Y_{2}=\gamma Y_{1}$ and $Z_{2}=\gamma Z_{1}$, then:

$$
\begin{aligned}
\tilde{\pi}_{i}\left(\left[X_{2}: Y_{2}: Z_{2}\right]\right)= & {\left[\pi_{i}\left(X_{2}\right): \pi_{i}\left(Y_{2}\right): \pi_{i}\left(Z_{2}\right)\right] } \\
= & {\left[\pi_{i}(\gamma) \pi_{i}\left(X_{1}\right): \pi_{i}(\gamma) \pi_{i}\left(Y_{1}\right): \pi_{i}(\gamma)\right.} \\
& \left.\pi_{i}\left(Z_{1}\right)\right] \\
= & {\left[\pi_{i}\left(X_{1}\right): \pi_{i}\left(Y_{1}\right): \pi_{i}\left(Z_{1}\right)\right] } \\
= & \pi_{i}\left(\left[X_{1}: Y_{1}: Z_{1}\right]\right) .
\end{aligned}
$$

\section{The classification of elements in $M_{A, B}\left(\mathbb{F}_{q}[e]\right)$}

Let $M_{A, B}\left(\mathbb{F}_{q}[e]\right)$ be the Montgomery curve $B Y^{2} Z=$ $X^{3}+A X^{2} Z+X Z^{2}$ over the ring $\mathbb{F}_{q}[e]$. In this section we will classify the elements of the Montgomery curves, into three types, depending on whether the projective coordinate $Z$ is invertible or not. The result is in the following proposition.

Proposition 3. The set $M_{A, B}\left(\mathbb{F}_{q}[e]\right)$ has the following form:

$$
\begin{aligned}
M_{A, B}\left(\mathbb{F}_{q}[e]\right) & =\left\{[X: Y: 1] \mid B Y^{2}=X^{3}+A X^{2}+X\right\} \\
& \cup\{[0: 1: 0]\} \\
& \cup\left\{[x e: 1: z e] \mid[x: 1: z] \in M_{\pi_{1}(A), \pi_{1}(B)}\right. \\
& \left.\left(\mathbb{F}_{q}\right)\right\} \cup\{[x e: y-y e: e] \mid[x: 0: 1] \in \\
& \left.M_{\pi_{1}(A), \pi_{1}(B)}\left(\mathbb{F}_{q}\right)\right\} \cup\{[x-x e: 1: z-z e] \mid \\
& {\left.[x: 1: z] \in M_{\pi_{0}(A), \pi_{0}(B)}\left(\mathbb{F}_{q}\right)\right\} \cup\{[x-x e:} \\
& \left.y e: 1-e] \mid[x: 0: 1] \in M_{\pi_{0}(A), \pi_{0}(B)}\left(\mathbb{F}_{q}\right)\right\} .
\end{aligned}
$$

Proof. Let $\left.P=[X: Y: Z] \in M_{A, B}\left(\mathbb{F}_{q}[e]\right)\right)$, where $X=x_{0}+x_{1} e, Y=y_{0}+y_{1} e$ and $Z=z_{0}+z_{1} e$.

We have two cases of the projective coordinate $\mathrm{Z}$ : 1) First case: $Z$ is invertible, then: $[X: Y: Z] \sim$ $[X: Y: 1]$, where $\sim$ is the equivalence relation of the projective space $P^{2}\left(\mathbb{F}_{q}[e]\right)[9$, p.6] (see also [1, 4, 6, 5, 7]).

2) Second case: $Z$ is no invertible, in this case we have:

i) $Z=z e$, where $z \in \mathbb{F}_{q}$, then:

- If $z=0$ then $[X: Y: Z]=[0: 1: 0]$, else $z \neq 0$ :

We have: $\pi_{0}\left(\left[x_{0}+x_{1} e: y_{0}+y_{1} e: z e\right]\right)=\left[x_{0}: y_{0}: 0\right] \in$ $M_{\pi_{0}(A), \pi_{0}(B)}$, then $x_{0}=0$ and $y_{0} \neq 0$, i.e:

$$
[X: Y: Z]=[x e: 1+y e: z e]
$$

there are two sub-cases of $y \in \mathbb{F}_{q}$ :

a) $y \neq-1$, then $1+y e$ is invertible in $\mathbb{F}_{q}[e]$, so we have: $[X: Y: Z] \sim[x e: 1: z e]$, where $[x: 1: z] \in$
$M_{\pi_{1}(A), \pi_{1}(B)}\left(\mathbb{F}_{q}\right)$.

b) $y=1$ then $1-e$ is not invertible in $\mathbb{F}_{q}[e]$, so we have: $[X: Y: Z]=[x e: 1-e: z e]$, where $[x: 1$ : $z] \in M_{\pi_{1}(A), \pi_{1}(B)}\left(\mathbb{F}_{q}\right)$ then necessary $z \neq 0$ according to Montgomery equation, hence $[X: Y: Z] \sim[x e$ : $y-y e: e]$, where $[x: 0: 1] \in M_{\pi_{1}(A), \pi_{1}(B)}\left(\mathbb{F}_{q}\right)$

ii) $Z=z-z e$, where $z \in \mathbb{F}_{q}$.

- If $z=0$ then $[X: Y: Z]=[0: 1: 0]$, else $z \neq 0$, we have $\pi_{1}\left(\left[x_{0}+x_{1} e: y_{0}+y_{1} e: z-z e\right]\right)=\left[x_{0}+x_{1}\right.$ : $\left.y_{0}+y_{1}: 0\right] \in M_{\pi_{1}(A), \pi_{1}(B)}$ then: $x_{0}+x_{1}=0$ and $y_{0}+$ $y_{1} \neq 0$, i.e:

$$
[X: Y: Z]=\left[x-x e: y_{0}+y_{1} e: z-z e\right]
$$

there are two sub-cases of $y_{0} \in \mathbb{F}_{q}$ :

a) $y_{0} \neq 0$ then $y_{0}+y_{1} e$ is invertible in $\mathbb{F}_{q}[e]$, so we have:

$$
[X: Y: Z] \sim[x-x e: 1: z-z e]
$$

b) $y_{0}=0$ then $y_{0}+y_{1} e$ is not invertible in $\mathbb{F}_{q}[e]$, so we have: $[X: Y: Z]=[x-x e: y e: z-z e]$, where $[x: 0: z] \in M_{\pi_{0}(A), \pi_{0}(B)}\left(\mathbb{F}_{q}\right)$ then necessary $z \neq 0$ according to Montgomery equation, hence $[X: Y: Z] \sim[x-x e: y e: 1-e]$, where $[x: 0: 1] \in$ $M_{\pi_{0}(A), \pi_{0}(B)}\left(\mathbb{F}_{q}\right)$

Corollary 4. $\tilde{\pi}_{0}$ is a surjective mapping.

Proof. Let $[x: y: z] \in M_{\pi_{0}(A), \pi_{0}(B)}\left(\mathbb{F}_{q}\right)$, then:

- if $y=0$ then $z \neq 0$ so $[x: y: z] \sim[x: 0: 1]$; hence $[x-x e: e: 1-e]$ is an antecedent of $[x: 0: z]$

- if $y \neq 0$, then $[x: y: z] \sim[x: 1: z]$; hence $[x-x e$ : $1: z-z e]$ is an antecedent of $[x: 1: z]$.

Corollary $5 . \tilde{\pi}_{1}$ is a surjective mapping.

Proof. Let $[x: y: z] \in M_{\pi_{1}(A), \pi_{1}(B)}\left(\mathbb{F}_{q}\right)$, then:

- if $y=0$ then $z \neq 0$ so $[x: y: z] \sim[x: 0: 1]$; hence $[x e: 1-e: e]$ is an antecedent of $[x: 0: 1]$

- if $y \neq 0$, then $[x: y: z] \sim[x: 1: z]$; hence $[x e: 1: z e]$ is an antecedent of $[x: 1: z]$.

The next proposition gives a bijection between the two sets $M_{A, B}\left(\mathbb{F}_{q}[e]\right)$ and $M_{\pi_{0}(A), \pi_{0}(B)}\left(\mathbb{F}_{q}\right) \times$ $M_{\pi_{1}(A), \pi_{1}(B)}\left(\mathbb{F}_{q}\right)$.

Proposition 4 . The $\tilde{\pi}$ mapping defined by:

$$
\begin{array}{rlcc}
\tilde{\pi}: M_{A, B}\left(\mathbb{F}_{q}[e]\right) & \rightarrow & M_{\pi_{0}(A), \pi_{0}(B)}\left(\mathbb{F}_{q}\right) \times \\
& & M_{\pi_{1}(A), \pi_{1}(B)}\left(\mathbb{F}_{q}\right) \\
{[X: Y: Z]} & \mapsto & \left(\left[\pi_{0}(X): \pi_{0}(Y): \pi_{0}(Z)\right],\right. \\
& & \left.\left[\pi_{1}(X): \pi_{1}(Y): \pi_{1}(Z)\right]\right)
\end{array}
$$

is a bijection.

Proof. • As $\tilde{\pi}_{0}$ and $\tilde{\pi}_{1}$ are well defined, then $\tilde{\pi}$ is well defined.

- Let $\left(\left[x_{0}: y_{0}: z_{0}\right],\left[x_{1}: y_{1}: z_{1}\right]\right) \in M_{\pi_{0}(A), \pi_{0}(B)}\left(\mathbb{F}_{q}\right) \times$ 
$\left.M_{\pi_{1}(A), \pi_{1}(B)}\left(\mathbb{F}_{q}\right)\right)$, clearly:

$\tilde{\pi}\left(\left[x_{0}+\left(x_{1}-x_{0}\right) e: y_{0}+\left(y_{1}-y_{0}\right) e: z_{0}+\left(z_{1}-z_{0}\right) e\right]\right)=$ $\left(\left[x_{0}: y_{0}: z_{0}\right],\left[x_{1}: y_{1}: z_{1}\right]\right)$, hence $\tilde{\pi}$ is a surjective mapping.

- Let $[X: Y: Z]$ and $\left[X^{\prime}: Y^{\prime}: Z^{\prime}\right]$ are elements of $M_{A, B}\left(\mathbb{F}_{q}[e]\right)$, where $X=x_{0}+x_{1} e, Y=y_{0}+y_{1} e, Z=$ $z_{0}+z_{1} e, X^{\prime}=x_{0}^{\prime}+x_{1}^{\prime} e, Y^{\prime}=y_{0}^{\prime}+y_{1}^{\prime} e$ and $Z^{\prime}=z_{0}^{\prime}+$ $z_{1}^{\prime} e$,

such that: $\tilde{\pi}([X: Y: Z])=\tilde{\pi}\left(\left[X^{\prime}: Y^{\prime}: Z^{\prime}\right]\right)$,

then:

$$
\left[x_{0}: y_{0}: z_{0}\right]=\left[x_{0}^{\prime}: y_{0}^{\prime}: z_{0}^{\prime}\right]
$$

and

$$
\left[x_{0}+x_{1}: y_{0}+y_{1}: z_{0}+z_{1}\right]=\left[x_{0}^{\prime}+x_{1}^{\prime}: y_{0}^{\prime}+y_{1}^{\prime}: z_{0}^{\prime}+z_{1}^{\prime}\right] \text {, }
$$

then there exist $(k, l) \in\left(\mathbb{F}_{q}^{*}\right)^{2}$ such that:

$\left\{\begin{array}{l}x_{0}^{\prime}=k x_{0} \\ y_{0}^{\prime}=k y_{0} \\ z_{0}^{\prime}=k z_{0}\end{array}\right.$ and $\left\{\begin{array}{l}x_{0}^{\prime}+x_{1}^{\prime}=l\left(x_{0}+x_{1}\right) \\ y_{0}^{\prime}+y_{1}^{\prime}=l\left(y_{0}+y_{1}\right) \\ z_{0}^{\prime}+z_{1}^{\prime}=l\left(z_{0}+z_{1}\right)\end{array}\right.$

So $\left\{\begin{array}{l}x_{1}^{\prime}=(l-k) x_{0}+x_{1} \\ y_{1}^{\prime}=(l-k) y_{0}+y_{1} \\ z_{1}^{\prime}=(l-k) z_{0}+z_{1}\end{array}\right.$

Then:

$\left\{\begin{array}{l}X^{\prime}=k x_{0}+\left((l-k) x_{0}+x_{1}\right) e=(k+(l-k) e) X \\ Y^{\prime}=k y_{0}+\left((l-k) y_{0}+y_{1}\right) e=(k+(l-k) e) Y \\ Z^{\prime}=k z_{0}+\left((l-k) z_{0}+z_{1}\right) e=(k+(l-k) e) Z\end{array}\right.$

As $k+(l-k) e$ is invertible in $\mathbb{F}_{q}[e]$, so $\left[X^{\prime}: Y^{\prime}: Z^{\prime}\right]=[X: Y: Z]$, hence $\tilde{\pi}$ is an injective mapping.

We can easily show that the mapping $\tilde{\pi}^{-1}$ defined by:

$$
\begin{gathered}
\tilde{\pi}^{-1}\left(\left[x_{0}: y_{0}: z_{0}\right],\left[x_{1}: y_{1}: z_{1}\right]\right)=\left[x_{0}+\left(x_{1}-x_{0}\right) e: y_{0}+\right. \\
\left.\left(y_{1}-y_{0}\right) e: z_{0}+\left(z_{1}-z_{0}\right) e\right]
\end{gathered}
$$

is the inverse of $\tilde{\pi}$.

Corollary 6. The cardinal of $M_{A, B}\left(\mathbb{F}_{q}[e]\right)$ is equal to the cardinal of $M_{\pi_{0}(A), \pi_{0}(B)}\left(\mathbb{F}_{q}\right) \times M_{\pi_{1}(A), \pi_{1}(B)}\left(\mathbb{F}_{q}\right)$.

\section{The group law}

Let $P=\left(X_{1}: Y_{1}: Z_{1}\right)$ be a point on $M_{A, B}\left(\mathbb{F}_{q}[e]\right)$ and $[n] P=\left(X_{n}: Y_{n}: Z_{n}\right)$. By 10 , the sum $[n+$ $m] P=[n] P \oplus[m] P$ is given by the following formulas where $Y_{n}$ never appears.

Addition: $n \neq m$

$$
\begin{aligned}
X_{m+n}= & Z_{m-n}\left(\left(X_{m}-Z_{m}\right)\left(X_{n}+Z_{n}\right)+\left(X_{m}+Z_{m}\right)\right. \\
& \left.\left(X_{n}-Z_{n}\right)\right)^{2}, \\
Z_{m+n}= & X_{m-n}\left(\left(X_{m}-Z_{m}\right)\left(X_{n}+Z_{n}\right)-\left(X_{m}+Z_{m}\right)\right. \\
& \left.\left(X_{n}-Z_{n}\right)\right)^{2} .
\end{aligned}
$$

Doubling: $n=m$

$$
\begin{aligned}
4 X_{n} Z_{n} & =\left(X_{n}+Z_{n}\right)^{2}-\left(X_{n}-Z_{n}\right)^{2}, \\
X_{2 n} & =\left(X_{n}+Z_{n}\right)^{2}\left(X_{n}-Z_{n}\right)^{2}, \\
Z_{2 n} & =4 X_{n} Z_{n}\left(\left(X_{n}-Z_{n}\right)^{2}+((A+2) / 4)\left(4 X_{n} Z_{n}\right)\right) .
\end{aligned}
$$

\section{Cryptography applications}

\subsection{Cryptography results}

From the proposition 4, we have:

- If $\operatorname{card}\left(M_{A ; B}\left(\mathbb{F}_{q}[e]\right)\right):=n$ is an odd number, then $n=s \times t$ is the factorization of $n$, where $s:=\operatorname{card}\left(M_{\pi_{0}(A) ; \pi_{0}(B)}\left(\mathbb{F}_{q}\right)\right)$ and $t:=$ $\operatorname{card}\left(M_{\pi_{1}(A) ; \pi_{1}(B)}\left(\mathbb{F}_{q}\right)\right)$, hence the cardinal of $M_{A ; B}\left(\mathbb{F}_{q}[e]\right)$ is not a prime number.

- The discrete logarithm problem in $M_{A, B}\left(\mathbb{F}_{q}[e]\right)$ is equivalent to the discrete logarithm problem in $M_{\pi_{0}(A) ; \pi_{0}(B)}\left(\mathbb{F}_{q}\right) \times M_{\pi_{1}(A) ; \pi_{1}(B)}\left(\mathbb{F}_{q}\right)$.

\subsection{ElGamal cryptosystem on a \\ Montgomery curves over this ring}

ElGamal cryptosystem for $M_{A, B}\left(\mathbb{F}_{q}[e]\right)$ consists essentially in mapping the operations customarily carried out in the multiplicative group $\mathbb{Z}_{p}$ to the set of points of a Montgomery curve $M_{A, B}\left(\mathbb{F}_{q}[e]\right)$, endowed with an additive group operation. An entity chooses and publishes a prime number $\mathrm{p}$ (large), a Montgomery curve $M_{A, B}\left(\mathbb{F}_{q}[e]\right)$ and a point $\mathrm{P}$ in $M_{A, B}\left(\mathbb{F}_{q}[e]\right)$.

\subsubsection{Key creation:}

- Choose a secret integer $s_{A}$.

- Compute $Q_{A}=s_{A} P$ in $M_{A, B}\left(\mathbb{F}_{q}[e]\right)$.

- Publish the public key $Q_{A}$.

\subsubsection{Encryption:}

- Choose the plain text $P_{m}$ in $M_{A, B}\left(\mathbb{F}_{q}[e]\right)$.

- Choose an ephemeral key $k$.

- Use Alice's public key $Q_{A}$ to calculate $u=k P$ in $M_{A, B}\left(\mathbb{F}_{q}[e]\right)$ and $v=P_{m}+k Q_{A}$ in $M_{A, B}\left(\mathbb{F}_{q}[e]\right)$.

- Send the cipher text (u,v)

\subsubsection{Decryption:}

Calculate $v-s_{A} u$ in $M_{A, B}\left(\mathbb{F}_{q}[e]\right)$. This value is equal to $P_{m}$.

ElGamal cryptosystem is directly based on the difficulty of solving the discrete logarithm problem over $(E,+)$ of base $P$. This problem requires to find $n$ where $Q=n P$ and points $P, Q$ belong to a set of points $E$ of a Montgomery curve $M_{A, B}\left(\mathbb{F}_{q}[e]\right)$. It is known to be computationally difficult and thus can be utilized to accomplish a more elevated level of security in cryptosystem. 
References:

[1] Ben Taleb, E.M., Chillali, A., El Fadil, L., Twisted Hessian curves over the Ring $\mathbb{F}_{q}[e], e^{2}=e$, Bol. Soc. Paran, (3s.) v.(40), doi:10.52699/bspm.15867, (2022).

[2] Boulbot, A., Chillali, A., Mouhib, A., "Elliptic Curves Over the Ring R", Bol. Soc. Paran., Vol.38, No.3, 2020, 193-201.

[3] ElGamal, T., A Public Key Cryptosystem and a Signature Scheme Based on Discrete Logarithms, In Proceedings of CRYPTO 84 on Advances in cryptology. Springer-Verlag New York, Inc, 1985, pp. 10-18.

[4] Grini, A., Chillali, A., Mouanis, H., The Binary Operations Calculus in $H_{a, d}^{2}$. Bol. Soc. Paran, Vol.40, 2020, 1-6.

[5] Grini, A., Chillali, A., Mouanis, H., Cryptography over twisted Hessian curves of the ring $F_{q}[\varepsilon], \varepsilon^{2}=0$. Adv. Math.: Sci. J., vol.10, no.1, 2021, 235-243.

[6] Grini, A., Chillali, A. \& Mouanis, H. A new cryptosystem based on a twisted Hessian curve $H_{a, d}^{4}$. J. Appl. Math. Comput., 2021.

[7] Grini A., Chillali A., Mouanis H. Cryptography Over the Twisted Hessian Curve $H_{a, d}^{3}$. In:
Ben Ahmed M., Teodorescu HN.L., Mazri T., Subashini P., Boudhir A.A. (eds) Networking, Intelligent Systems and Security. Smart Innovation, Systems and Technologies, vol. 237. Springer, Singapore, 2022.

[8] Hendrik, W., Lenstra Jr., Factoring integers with elliptic curves. Annals of mathe-matics, 1987, pp. 649-673.

[9] Lenstra, H. W., Eliptic Curves and NumberTheoretic Algorithms. Processing Int. Congress Math., USA, 1986.

[10] Peter L., Montgomery, Speeding the Pollard and Elliptic Curve Methods of Facorization, Mathematics of Computation., vol. 48, 1987, 243-264.

\section{Creative Commons Attribution}

License 4.0 (Attribution 4.0

International , CC BY 4.0)

This article is published under the terms of the Creative Commons Attribution License 4.0

https://creativecommons.org/licenses/by/4.0/deed.en_US 